\title{
Safety Risk Evaluation on Mobile Payment Based on Improved AHP Method
}

\author{
Yang Zhang ${ }^{1, a)}$, Yong Shao ${ }^{1, b)}$, Chang shun Yan ${ }^{1, c)}$, Yue Zhou ${ }^{2, d)}$ and \\ Xiujun Wang ${ }^{2, \mathrm{e})}$ \\ ${ }^{1}$ Department of Informatics, Beijing University of Technology, Beijing, 100124, China. \\ ${ }^{2}$ Beijing Software Product Quality Testing Center, Beijing100193, China \\ a) Corresponding author: 15600520022@163.com \\ b) shaoyong@bjut.edu.cn \\ c)yuewuxing@bjut.edu.cn \\ d) $1241562483 @ q q . c o m$ \\ e) mazels@126.com
}

\begin{abstract}
Mobile payment is a very important service in this age of the Internet, but threats from a safety perspective are very serious. Based on the uniqueness of the mobile payment, establishes a hierarchy appraisal target system by improving the AHP method. To better determine the weight that affects the safety risk factors for mobile payment, it adopts a 3-scale method to replace the traditional 9-scale method in the construction of AHP judgment matrix and then compare it with the traditional 9-scale method. The comparison results demonstrate that the improved method does overcome the difficulty of the expert grasping the scale of judgment in the traditional analytic hierarchy process. The evaluation index structure and the adoption of improved AHP method can provide effective security risk assessment for mobile payment, can improve the efficiency and accuracy of mobile payment security risk assessment, and has strong practicality.
\end{abstract}

Key words: Mobile payment; Analytic hierarchy process; Improved AHP method; Safety risk learning.

\section{INTRODUCTION}

Along with development of smart phone and mobile internet and internet of things, mobile payment is involving into our everyday life more deeply. Data from China Third Party Mobile Payment Market Quarterly Monitoring Report of Third Quarter of 2017 indicates, the transaction size for the china third party payment had reached 29495.92 billion Yuan in the first quarter of 2017, with year on year increasing $28.02 \%$. So, China has become the biggest mobile payment market in the world.

Despite the convenience that the mobile payment is brought to us, it also has many risk problems. The 2016 Mobile Payment User Investigation Report indicates people are most concerned about the safety problem on mobile payment. And the 2016 Mobile Payment Safety Investigation Report issued by China UnionPay also displays that the mobile payment is more vulnerable to the threat of the outlaws, with many safety risk problems. To attract user quickly, some of payment applications look down upon the safety risk. There are many safety problems on mobile payment, take the example of intelligence POS (Point of Sale) security hole which is exposed in the CCTV evening party of 2016; Suzhou Alipay 1999 Yuan group identity theft event, and ETC (Electronic Toll Collection) Cobranded card identity theft event. This indicates the safety risk is ubiquitous in each link of the mobile payment industry china, which will impose threat to the safety of our property safety personal information. Thus, it is necessary to perform safety risk evaluation to the mobile payment. 
From domestic and overseas research status, the researchers at home and abroad have made some progress on the safety risk assessment method. Such as Dong Xiaoning [1] et al. Put forward a risk assessment method based on fuzzy mathematics theory and evidence theory, which provides powerful data for risk control and security defense of information system support. Fu Chunchuan [2] et al. In view of the information security risk of Train Control Center (TCC), put forward a column-based risk assessment method based on CMOSA (Component Model Based on Security Attributes) Risk and it provides reference for risk managers to identify unit risks and manage them effectively. Liu Jinghui [3] adopted a combination of Fault Tree Analysis(FTA) and Analytic Hierarchy Process (AHP) to study and design a comprehensive evaluation method of railway security risk based on FTA-AHP, so as to reflect the security risk of the system status more comprehensively. Semin [4] and others discussed the issue of information system security threat assessment, noted the disadvantages of traditional approaches to threat assessment and proposed an alternative approach based on event statistics. Treetippayaruk [5] et al. Compared the severity of the vulnerabilities caused by the upgrade with the vulnerabilities of the currently installed software and proposed a security vulnerability assessment method based on CVSS (Common Vulnerability Scoring System) vulnerability scoring system to indicate whether the upgrade Really improve the safety. Beomjun Kim [6] and others proposed a method of primal probability threat assessment to predict and avoid collisions that may occur in multivehicle traffic and verify the overall performance of the proposed threat assessment algorithm through actual road vehicle testing. Although some progresses have been made in the research of safety risk assessment methods, few studies have been conducted on the assessment methods of safety risk in mobile payment.

Currently, there are two kinds of research on the security of mobile payment. One is focus on analyzing on safety problem and give prevention measures on the mobile payment [7-10]; the other is stressed on mobile payment risk evaluation method and evaluation model [11-12]. The latter is aimed to establish a reasonable risk evaluation method by analyzing the main risk of the mobile payment. Most of the risk evaluation models are established based on the traditional hierarchy method and expert knowledge. However, there are some shortcomings for this method: firstly, the 1-9 scale method used to establish judgment matrix is much rough and is hard to compare the relative importance between the two objects; secondly, the traditional method needs the consistent measurement, whose whole process is very complicated without existing rule to follow. As for its limitations, some researchers have made corresponding improvements to the algorithm. For example, O. Yu has developed a set of system programs for modifying matrix inconsistencies that provide a simplified and easy-to-use standard for consistency of judgment matrices in AHP [13]. Pankratova N proposed a method without experts' participation to improve the consistency of judgment matrix. It was verified that the coefficients of judgment matrix consistency were not always able to correctly evaluate the consistency of the matrix. In order to judge the consistency of the matrix more reliably, some other attributes should be used to reduce the Inconsistency in the judgment matrix [14]. Most researchers only make simple improvements and adjustments to the complicated problem of matrix consistency checking. Although the problem has been alleviated somewhat, the problem has not been solved fundamentally. Therefore, this paper presents a weight calculation method without matrix consistency checking, so as to avoid the complex problem of matrix consistency checking and make the risk assessment process easier. By analyzing the consistency of the safety risk for the mobile payment safety, the article divides the mobile payment safety risk into three layers: target layer, feature layer and factor layer, and establishes the corresponding index system, then invite experts to evaluate each safety factor. By computing weight of each factor and using three scale method to build comparative judgment matrix, it is verified that the improved AHP method can better overcome the subjectivity and fuzziness of human judgment to the assessment and calculate the conclusion that is simple and does not need consistency test.

\section{SAFETY RISK ANALYSIS ON MOBILE PAYMENT}

\section{Threats to Mobile Payment}

There are many ways to classify mobile payment. According to the interactive process of payment, mobile payment can be divided into near-field payment and remote payment. Near-field payment refers to a kind of payment through the mobile terminal using near field wireless communication technology when consumers buy goods or services. The use of mainstream technologies includes Radio Frequency Identification(RFID), infrared, Bluetooth, etc. such as the cell phone public traffic card. The remote payment realizes all kinds of transfer and consumption function through interaction between mobile network and background server, can be divided into mobile Internet payment and SMS payment, the use of the mainstream technology including information technology and data communication technology, etc., such as buying things in Taobao. This paper combines near-field payment 
and remote payment of these two payment methods and the 2016 Mobile Safety Annual Report and the several accidents happened for the mobile payment in recent years, and through consultation with relevant experts in the field, to conclude that the current mobile Payment security threats include the following aspects.

Threats from the Software

Software attack includes loophole coming from operational system and application software. As an open platform, the operational system is more likely to be attacked from design, such as stealing account, theft of privacy, calling and sending short message malicious act, which bring all kinds of safety threat to the user. For the application software developed on the basis of operational system, malicious code is an important mature threat method that has not brought enough attention to the people. The much common software attack mainly includes phishing attack, man in the middle attack, sensitive data tapping, transaction manipulation, transaction forge, and threat form false base station.

Threats from Communication Protocol

For the communication protocol, the attack can be performed through sniffers, intercept, interpolate, forging transmit information and forging identity information. Such the vulnerability of communication protocol, disturbed communication and delayed communication capability, all of which may threaten the security of mobile payment.

Threats from Hardware

The hardware attack includes two aspects: one is basic hardware attack, and the other is SE (Security Element) attack. The basic hardware safety threat consists of bus listening, calling debug interface and use simple tools to attack, while the SE advanced hardware attack includes hardware chip attack utilizing time, power consumption, electromagnetic radiation, and fault injection as well as special tools to attack.

Threats from Other Aspects

For the safety of sweeping card, the personal identity information such as payment account and password may be stolen after sweeping; for the free Wi-Fi hotspot, the privacy is likely to be disclosed as a result of the low safety of public Wi-Fi; The NFC (Near Field Communication) mobile payment has safety problems of tapping, tampering with data and man in the middle attack; the third-party payment platform has safety problems such as money laundering, credit cashing, precipitation funds.

\section{Safety Target for the Mobile Payment}

As a popular payment method, the mobile payment should not only meet the safety requirements of the internet, but also should meet more advanced safety requirement. Generally speaking, the mobile payment should differ from the general internet payment on the following aspects:

With small storage and weak computing power, the mobile terminal is not easy to use high intensity asymmetry encryption algorithm.

Mobile terminal software is not powerful enough, its security is relatively poor compared to the computer, such as its browser cannot support the password control and other controls.

With low scalability, it is hard to apply USB key and digital certificate

The openness of mobile terminals makes them more vulnerable to malicious attacks. For example, mobile networks are more open than wired networks. Mobile networks transmit wireless signals without definitive defensive boundaries and are easily attacked by attackers. Moreover, mobile networks are susceptible to interference and can cause large fluctuations in signal quality, it will also be disturbed resulting to communication problem.

So, the aim of the research is to establish safety risk indicator system for the mobile payment, calculate the relative importance of each impact index through improved AHP and find the factor that affects the safety of mobile payment mostly, thus provide reference for the safety prevention for the mobile payment in the big data times.

\section{Improved Analytic Hierarchy Process}

\section{Analytic Hierarchy Process}

Analytic hierarchy process, shortened as AHP, is first proposed by the America operational research T.L. Satty. It is a systematic and hierarchical method by combining the qualitative and quantities. It is a combination of qualitative and quantitative, systematic, hierarchical analysis. The main idea of this method is firstly to stratify complex problems reasonably and then compare and judge the degree of importance between the two indexes. 
Introducing the 1-9 scale method to establish the judgment matrix and calculating the eigenvectors of the judgment matrix for the purpose of providing basis for selecting the important indicator.

There are many disadvantages for the traditional 1-9 scale method, one is the possibility of unreasonable judgment matrix as a result of subjectivity and sidedness by the experts; another is complexity of matrix consistent measurement and large computation which are liable to bias the judgment results.

The improved hierarchy process builds the judgment matrix using 1,0,-1 three scales to compare the factors in each layer, that is, compare indicator 1 and indicator 2 , if indicator 1 is important than indicator 2 , then indicate with 1 ; if indicator 1 is equal to indicator 2 , then indicate with 0 ; if indicator 1 is less important than indicator 2 , then indicate with -1. Three-scale method can make experts easily to make an accurate judgment on the relative importance of each two factors, does not require the consistency checking, and the computing process simpler.

\section{Steps of Improving Analytic Hierarchy Process}

Step 1: Establish hierarchy model for the problem

Step 2: Determine the comparison judgment matrix. Divide the evaluation standard into three layers using three scale methods: 1,0 and -1 . Assume the judgment matrix built by the standard is $A=\left(a_{i j}\right)_{n \times n}$, then:

$$
\mathrm{A}=\left[\begin{array}{cccc}
\mathrm{a}_{11} & \mathrm{a}_{12} & \cdots & \mathrm{a}_{1 \mathrm{n}} \\
\mathrm{a}_{21} & \mathrm{a}_{22} & \cdots & \mathrm{a}_{2 \mathrm{n}} \\
\vdots & \vdots & \vdots & \vdots \\
\mathrm{a}_{\mathrm{n} 1} & \mathrm{a}_{\mathrm{n} 2} & \cdots & \mathrm{a}_{\mathrm{nn}}
\end{array}\right]
$$

Among which

$$
a_{i j}=\left\{\begin{array}{cc}
1 & 1 \text { indicates indicator } \mathrm{i} \text { is more important than indicator } \mathrm{j} \\
0 \quad 0 \text { indicates indicator } \mathrm{I} \text { is equal to the indicator } \mathrm{j} \\
-1 \quad-1 \text { indicates indicator } \mathrm{j} \text { is more important than indicator } \mathrm{i}
\end{array}\right.
$$

Step 3: Determine optimal transfer matrix. Set optimal transfer matrix of matrix A is $\mathrm{O}=\left(\mathrm{o}_{\mathrm{ij}}\right)_{\mathrm{nxn}}$, then:

$$
\mathrm{O}=\left[\begin{array}{cccc}
\mathrm{o}_{11} & \mathrm{o}_{12} & \cdots & \mathrm{o}_{1 \mathrm{n}} \\
\mathrm{o}_{21} & \mathrm{o}_{22} & \cdots & \mathrm{o}_{2 \mathrm{n}} \\
\vdots & \vdots & \vdots & \vdots \\
\mathrm{o}_{\mathrm{n} 1} & \mathrm{o}_{\mathrm{n} 2} & \cdots & \mathrm{o}_{\mathrm{nn}}
\end{array}\right]
$$

Among which

$$
\mathrm{o}_{\mathrm{ij}}=\frac{1}{\mathrm{n}} \sum_{1=1}^{\mathrm{n}}\left(\mathrm{a}_{\mathrm{il}}+\mathrm{a}_{\mathrm{lj}}\right)
$$

Step 4: Determine optimal consistent matrix. Set optimal consistent matrix of matrix $\mathrm{O}$ is $\mathrm{C}=\left(\mathrm{c}_{\mathrm{ij}}\right)_{\mathrm{nxn}}$, then: 


$$
\mathrm{C}=\left[\begin{array}{cccc}
\mathrm{c}_{11} & \mathrm{c}_{12} & \cdots & \mathrm{c}_{1 \mathrm{n}} \\
\mathrm{c}_{21} & \mathrm{c}_{22} & \cdots & \mathrm{c}_{2 \mathrm{n}} \\
\vdots & \vdots & \vdots & \vdots \\
\mathrm{c}_{\mathrm{n} 1} & \mathrm{c}_{\mathrm{n} 2} & \cdots & \mathrm{c}_{\mathrm{nn}}
\end{array}\right]
$$

Along which

$$
\mathrm{c}_{\mathrm{ij}}=\exp \left(\mathrm{o}_{\mathrm{ij}}\right)
$$

Step 5: Determine relative weight for the corresponding indicating factor, set $W=\left\{w_{1}, w_{2}, \cdots, w_{n}\right\}$, then:

$$
\mathrm{w}_{\mathrm{i}}=\frac{\overline{\mathrm{w}_{\mathrm{i}}}}{\sum_{\mathrm{j}=1}^{\mathrm{n}} \overline{\mathrm{w}_{j}}}
$$

Along which

$$
\overline{\mathrm{w}_{\mathrm{i}}}=\left(\prod_{\mathrm{k}=1}^{\mathrm{n}} \mathrm{c}_{\mathrm{ik}}\right)^{1 / \mathrm{n}}
$$

\section{APPLICATION OF IMPROVED ANALYTIC HIERARCHY PROCESS IN THE MOBILE PAYMENT RISK EVALUATION}

\section{Establish Hierarchy Structure for Factors Influencing Mobile Payment Safety Risk}

By sorting out the mobile payment security problems that have appeared on people in recent years, identifying and analyzing the security risk factors of mobile payment and combining with the opinions of experts. Establish the analytic hierarchy structure for factors influencing mobile payment safety risk, displayed as Fig.1:

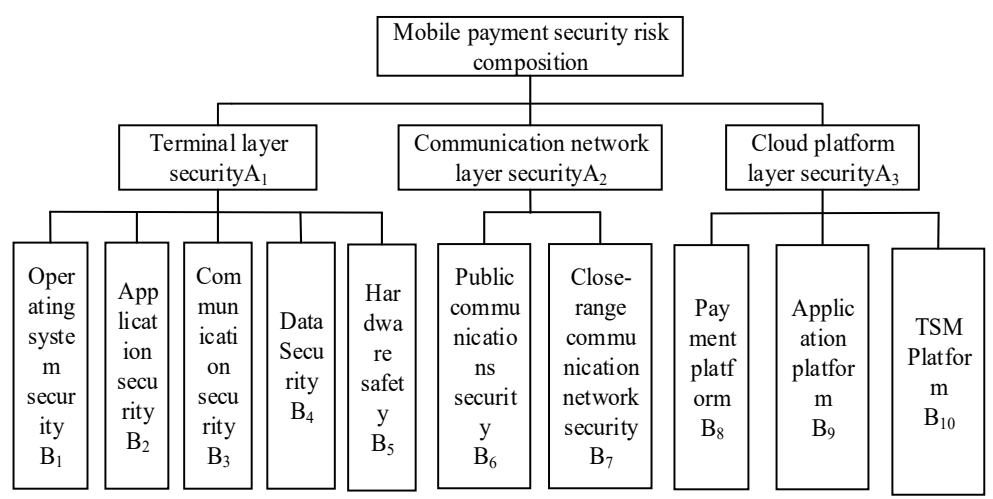

FIGURE 1. Hierarchy Model for Mobile Payment Risk Factors. 


\section{Determine Weight of Mobile Payment Risk Factor Indicator}

Take the example of secondary indicator weight. Base on the thinking of improved analytic hierarchy process, ten experts conclude a comparative matrix A using three scale methods to compare the importance of pair factors, that is:

$$
A=\left[\begin{array}{ccc}
0 & 1 & 1 \\
-1 & 0 & 1 \\
-1 & -1 & 0
\end{array}\right]
$$

Transform matrix A into optimal transfer matrix $\mathrm{O}$

$$
\text { That is: } O=\left[\begin{array}{ccc}
0 & \frac{2}{3} & \frac{4}{3} \\
-\frac{2}{3} & 0 & \frac{2}{3} \\
-\frac{4}{3} & -\frac{2}{3} & 0
\end{array}\right]
$$

Then transform the optimal transfer matrix into the optimal consistent matrix $\mathrm{C}$ that is:

$$
\mathrm{C}=\left[\begin{array}{lll}
1.0000 & 1.9477 & 3.7937 \\
0.5134 & 1.0000 & 1.9477 \\
0.2636 & 0.5134 & 1.0000
\end{array}\right]
$$

Get the relative weight of each secondary indicator by square root, that is $\mathrm{W}=(0.5627,0.2890,0.1483)$

From the results of $\mathrm{W}$, we can see, the weight for indicator A1, A2, A3 is $56.27 \%, 28.90 \%, 14.83 \%$ respectively. Similarly, the relative importance for each third level factor indicator is shown in Table 1.

TABLE 1. General Order for the Weight of Mobile Payment Safety Risk Factors.

\begin{tabular}{cccccc}
\hline B & A1 & A2 & A3 & Weight & Order \\
\hline B1 & 0.3222 & & 0.1989 & 1 \\
B2 & 0.3222 & & 0.1989 & 2 \\
B3 & 0.1185 & & 0.1207 & 3 \\
B4 & 0.1185 & & 0.0662 & 7 \\
B5 & 0.1185 & & 0.0662 & 8 \\
B6 & & 0.7311 & & 0.0809 & 4 \\
B7 & 0.2689 & & 0.0809 & 5 \\
B8 & & 0.4223 & 0.0731 & 6 \\
B9 & & 0.1554 & 0.0542 & 10 \\
B10 & & 0.4223 & 0.0600 & 9 \\
\hline
\end{tabular}

\section{The Improved AHP Method Compared with the Traditional AHP Method}

Because of the limited space of the article, taking the determination of the second-level indicator weight as an example, comparing the improved AHP method with the traditional hierarchical analysis method. According to the main idea of traditional AHP analysis method, experts are asked to compare the importance of two factors by 1-9 scale method.

Step1: Use the same level model as the one in Figure; 
Step2: Synthesize the judgment of five experts and construct the square matrix of $\mathrm{N}$ according to the fuzzy Delphi method. Analytic Hierarchy Process Consistency Test Calculation Method: Calculate the maximum eigenvalue $\lambda_{\max }$ and consistency index of judgment matrix:

$$
C l=\frac{\lambda \max -n}{n-1}
$$

Then calculate the proportion of consistency:

$$
C R=\frac{C l}{R I}
$$

$\mathrm{RI}$ is the average random consistency indicator and it related to $\mathrm{n}$; When, then it is acceptable to consider that the judgment matrix satisfies "consistency"; otherwise, the judgment matrix needs to be reconstructed or the proper correction is made.

$$
N=\left[\begin{array}{ccc}
1 & 2 & 3 \\
1 / 2 & 1 & 1 \\
1 / 3 & 1 & 1
\end{array}\right]
$$

Using the method of calculating the eigenvalues of the matrix, get the maximum eigenvalue is $\lambda_{\max }=3.0183, \mathrm{CI}=0.00915$, because when $\mathrm{n}=3, \mathrm{RI}=0.58$ (available from the table of $\mathrm{n}$ and $\mathrm{RI}$ values), $\mathrm{CR}=$ $0.015<0.1$, indicating that the constructed judgment matrix satisfies "consistency".

Step3: level single sorting. The eigenvector corresponding to the largest eigenvalue of the judgment matrix $\mathrm{N}$ is obtained. And after normalization, the weight vectors $\mathrm{W}=(0.4434,0.3874,0,1692)$ of the second-level index elements A1, A2 and A3 relative to the total target can be obtained.

Establish an improved AHP method and the traditional hierarchical method comparison table, as shown in Table 2.

So, the aim of the research is to establish safety risk indicator system for the mobile payment, calculate the relative importance of each impact index through improved AHP and find the factor that affects the safety of mobile payment mostly, thus provide reference for the safety prevention for the mobile payment in the big data times.

TABLE 2. The comparison table of Improves AHP method and Traditional hierarchical method.

\begin{tabular}{cc}
\hline & Improved AHP method \\
\hline $\mathrm{W}$ & $(0.5627,0.2890,0.1483)$ \\
Sort & $\mathrm{A} 1>\mathrm{A} 2>\mathrm{A} 3$ \\
\hline $\mathrm{W}$ & Traditional AHP method \\
\hline Sort & $(0.4434,0.3874,0,1692)$ \\
& $\mathrm{A} 1>\mathrm{A} 2>\mathrm{A} 3$ \\
\hline
\end{tabular}

The comparison results show that the improved AHP method is consistent with the traditional AHP method, which shows that the judgment matrix established by the 3-scale method and the method of conversion to the optimal consistency matrix can replace the consistency inspection process in the traditional AHP method. Compared with the maximum eigenvalue method of the traditional AHP method, using the improved AHP method to obtain the index weight is more simple and easy to operate. And if the judgment matrix of the traditional AHP method does not pass the consistency test, the judgment matrix needs to be reconstructed with a large amount of calculation and has a blindness. Sometimes after repeated rebuilds, the matrix may still not pass the one-time test to make the consistency check process complicated. However, using the improved AHP method not only eliminates the cumbersome consistency check process but also greatly reduces the calculation and the computational complexity. 


\section{RESULTS ANALYSIS}

After comparing the improved AHP method with the traditional AHP method, it not only verifies the operability, efficiency, superiority and practicability of the three-scale method, but also shows through analysis and calculation, we can see the most influencing factors for the mobile payment safety risks are operational system safety, application system safety, and communication safety and communication network safety. So, to ensure the safety of the mobile payment, we should enhance research on mobile terminal operational system and application safety, as well as communication safety and communication network safety. We have adopted many measures and prevention technology to counter the risk, such as on the software aspect, perform service logic safety analysis to avoid logic loophole, and integrate safety into the product life cycle, and establish perfect safety design, development, test and response process; on the hardware, protect the debugging interface to prevent unauthorized access; on the communication protocol, add safety certificate and access control mechanism to encrypt the transmit information, and research high safety solution. But the research is not enough. As a future payment method, the research on the mobile payment is very important. Only by solving the safety problem, will the user use the mobile payment tool with a relieved heart, and can the mobile payment develop more quickly.

\section{ACKNOWLEDGMENTS}

Foundation Items(No.2017YFF0209602): Software Testing Technology Beijing Key Laboratory: Research on Key Technology of NQI Resource Aggregation and Sharing--National Key Research and Development Program "Internet +" NQI Integrated Services Common Technologies Research Project.

\section{REFERENCES}

1. X.N. Dong, H.R. Zhao, D. W. Li, et al, Research on information system security risk assessment based on fuzzy evidence theory[J]. Information network security, 2017, (05):69-73.

2. C.C. Fu, X.M. Wang, W.F. Zhang, et al, Information security risk assessment method of the control center based on component safety properties [J], Journal of railway, 2017, 39(08):77-84.

3. J. H. Liu, Comprehensive evaluation method of railway safety risk based on FTA-AHP [J]. China railway science,2017, 38(2):138-144.

4. V.G. Semin, A. S. Kabanov, A. B. Los, A statistical approach to the assessment of security threats information system[C]//2017 International Conference "Quality Management, Transport and Information Security, Information Technologies” (IT\&QM\&IS), St. Petersburg, 2017:100-105.

5. S. Treetippayaruk, T. Senivongse, S. Treetippayaruk, T. Senivongse, Security vulnerability assessment for software version upgrade[C] //Ieee/acis International Conference on Software Engineering, Artificial Intelligence, NETWORKING and Parallel/distributed Computing.IEEE,2017:283-289.

6. Kim B, Park K, Yi K, Probabilistic Threat Assessment with Environment Description and Rule-based MultiTraffic Prediction for Integrated Risk Management System[J]. IEEE Intelligent Transportation Systems Magazine, 2017, 9(3):8-22.

7. Y.L. Liu, Z.G. Jin, T. Y. Gao, Research on safety of mobile payment system [J]. Information network security, 2017, (02):1-5.

8. Sung S, Kong E, Youn C, Mobile Payment Based on Transaction Certificate Using Cloud Self - Proxy Server[J]. Etri Journal, 2016, 39(1).

9. Kier C, Madlmayr G, Nawratil A, et al, Mobile Payment Fraud: A Practical View on the Technical Architecture and Starting Points for Forensic Analysis of New Attack Scenarios[C]// Ninth International Conference on It Security Incident Management \& It Forensics. IEEE, 2015:68-76.

10. Y.Q. Zhang, Z.Q. Wang, Q. X. Liu, et al, Progress and development of safety research of near-field communication technology [J]. Journal of computer science, 2016,39(06):1190-1207.

11. C.Lv, Quality Evaluation of English Teaching Class Based on FAHP[C]//International Conference on Intelligent Transportation, Big Data \& Smart City. IEEE Computer Society, 2016:241-244.

12. Y. Tian, X. Sun, Risk evaluation index system of navigation environment of Qiongzhou Strait based on FAHP[C]//International Conference on Transportation Information and Safety. IEEE, 2015:729-731. 
13. Yu. O, "Assessing and Improving Consistency of a Pairwise Comparison Matrix in the Analytic Hierarchy Process,"2017 Portland International Conference on Management of Engineering and Technology (PICMET), Portland, OR, 2017, pp. 1-6.

14. Pankratova N, and Nedashkovskaya N," Methods of Evaluation and Improvement of Consistency of Expert Pairwise Comparison Judgments", International Journal of Information Theories and Applications, vol. 22, pp. 203-223, 2015. 